\section{Are the short-term cost savings and benefits of an early psychosis program maintained at 8-year follow-up?}

\author{
MG Harris', C Mihalopoulos' ${ }^{2}$ LP Henry', SM \\ Harrigan', SF Farrelly', OS Schwartz', AL Prosser', \\ HJ Jackson ${ }^{1,3}$, PD McGorry ${ }^{1}$
}

'Department of Psychiatry, The University of Melbourne, Melbourne, Victoria, Australia; ${ }^{2}$ School of Population Health, The University of Melbourne, Melbourne, Victoria, Australia; and ${ }^{3}$ Department of Psychology, The University of Melbourne, Melbourne, Victoria, Australia

Background: Despite considerable interest and investment in early psychosis services over the past one to two decades, scant attention has been paid to the economic evaluation of such services. A 1-year evaluation of the cost-effectiveness of the Early Psychosis Prevention \& Intervention Centre (EPPIC) model in Melbourne, Australia, concluded that EPPIC was a dominant intervention compared with historical care in that it was cheaper and more effective; however, no published studies have evaluated the longer term effects of a model of early intervention in terms of both outcomes and costs. This study aims to examine whether the cost savings and benefits associated with EPPIC persist beyond the 1-year timeframe.

Methods: The study used a historical control design. A sample of 51 participants who presented to EPPIC in 1993 was individually matched (on age, sex, diagnosis, premorbid adjustment and marital status) with 51 participants admitted to the precursor service (the "preEPPIC' service) between 1989 and 1992. Participants were followed up at 1 year, then again approximately 8 years after inception. A representative subsample of 65 participants was interviewed at 8-year follow-up. Data describing psychiatric service use, medication type, duration and dosage were collected by means of interviews with patients and informants, electronic databases and medical records. Standard economic methods will be used to evaluate the two interventions.

Results: The results will compare the costs, benefits and incremental cost-effectiveness of the two interventions.

Conclusion: This study will help answer whether the EPPIC model of care maintains 'value for money' over a longer period.

\section{Cost-effectiveness of a preventive intervention for young people at ultrahigh risk of developing psychosis}

\author{
L Phillips', S Cotton'2, P McGorry², H Yuen², \\ C Mihalopoulos ${ }^{3}$, S Shih ${ }^{3}$, D Kelly', J Ward', \\ R Carter ${ }^{3}$, A Yung ${ }^{2}$
}

'Department of Psychology, The University of Melbourne, Melbourne, Victoria, Australia; ${ }^{2}$ Department of Psychiatry, The University of Melbourne, Melbourne, Victoria, Australia; and ${ }^{3}$ School of Population Health, The University of Melbourne, Melbourne, Victoria, Australia

Background: Recent research has shown that the provision of specific treatment to young people identified as being at 'ultrahigh risk' (UHR) of developing a psychotic disorder may delay, or even prevent, the onset of disorder. However, there are obvious costs associated with identifying and treating UHR individuals and whether such intervention provides value for money is important in the context of scarce health resources. This study aimed to determine the health sector costs associated with a randomized controlled trial for UHR participants attending the PACE Clinic in Melbourne, Australia. A second aim was to determine whether specific preventive intervention (SPI) resulted in cost savings over short (12 months) and long (12-36 month) follow-up periods.

Methods: Treatment was either an SPI (neuroleptic medication and cognitively oriented psychotherapy) or needs-based intervention (NBI; supportive psychotherapy alone).

Results: During the treatment phase, the SPI group incurred significantly higher therapy and total costs compared with the NBI group, but hospital and medication costs did not differ between the groups. There were no significant treatment cost differences between the SPI and NBI groups over the first follow-up phase. However, over the second follow-up phase, the SPI group incurred significantly lower therapy and total costs. Members of the NBI group who did not develop psychosis incurred significantly higher therapy and total costs compared with the SPI subgroup members who did not develop psychosis. There were no significant cost differences in treatment of the psychotic subgroups of the NBI and SPI groups over the long-term follow-up period.

Conclusion: This preliminary study has shown the long-term cost savings associated with specific treatment for young people at UHR for psychosis. 